\title{
Onset threshold of the plasma levels of soluble fms-like tyrosine kinase 1/placental growth factor ratio for predicting the imminent onset of preeclampsia within 4 weeks after blood sampling at 19-31 weeks of gestation
}

\author{
Akihide Ohkuchi ${ }^{1}$, Chikako Hirashima ${ }^{1}$, Kayo Takahashi, Hirotada Suzuki, Shigeki Matsubara and \\ Mitsuaki Suzuki
}

Our aim was to evaluate the onset threshold of plasma levels of the soluble fms-like tyrosine kinase 1 (sFlt-1)/placental growth factor (PIGF) ratio for predicting the imminent onset of preeclampsia (PE) within 4 weeks after blood sampling. We prospectively measured the plasma levels of sFIt-1 and PIGF by an automated electrochemiluminescence immunoassay at 19-25 weeks of gestation in 1199 women and at 26-31 weeks of gestation in 798 women. The onset threshold of the sFIt-1/PIGF ratio was determined as the 2.5th percentile of the 95th confidence interval $(\mathrm{CI})$ of a regression line between the onset gestational weeks of PE and the standard deviation score of $\log _{10}$ (sFIt-1/PIGF), using 25 samples taken within 1 week after the onset of PE. The imminent onset of PE was identified in $2(0.2 \%)$ women recruited at $19-25$ weeks and in $6(0.8 \%)$ women recruited at 26-31 weeks. The onset threshold of plasma levels of the sFIt-1/PIGF ratio at 19-25 weeks showed a sensitivity (SE) of 1.00 , a specificity (SP) of 1.00 , a positive likelihood ratio (LR + ) of $\infty$ and a positive predictive value (PPV) of 1.00; the onset threshold of plasma levels of the sFlt-1/PIGF ratio at 26-31 weeks showed a SE of 0.83, a SP of 0.994, a LR + of 132 (95\% Cl: 51-339) and a PPV of 0.50 . In conclusion, the onset threshold of plasma levels of the sFlt-1/PIGF ratio was shown to be a highly sensitive and a highly specific screening method for detecting the imminent onset of PE within 4 weeks after blood sampling at 19-31 weeks.

Hypertension Research (2013) 36, 1073-1080; doi:10.1038/hr.2013.95; published online 22 August 2013

Keywords: imminent onset; onset threshold; placental growth factor; preeclampsia; soluble fms-like tyrosine kinase 1

\section{INTRODUCTION}

Preeclampsia (PE) is a major complication of pregnancy associated with significant morbidity and mortality of both the fetus and the mother. ${ }^{1,2}$ Almost $3.5 \%$ of primiparous women and $2.0 \%$ of multiparous women in Japan develop PE. ${ }^{3}$ Recent years have seen considerable advances in the identification of biomarkers related to the development of PE, especially anti-angiogenic factors such as soluble fms-like tyrosine kinase 1 (sFlt-1) and soluble endoglin (sEng) and the circulating targets of these anti-angiogenic factors such as vascular endothelial growth factor, placental growth factor (PlGF) and transforming growth factor beta. ${ }^{4-9}$ Basic research using a pregnant rat/mouse model of PE using adenoviral vectors containing sFlt-1 and/or sEng has clearly substantiated the mechanism underlying the role of anti-angiogenic factors in the pathophysiology of PE. ${ }^{10-13}$ Despite the accumulation of evidence of the important roles of antiangiogenic factors in the genesis of $\mathrm{PE}$, the translation of these findings into clinically useful paradigms has moved at a slow pace. ${ }^{8}$ Indeed, no single biomarker except for the onset threshold of the sFlt1/PlGF ratio has fulfilled the criteria of clinically useful screening of PE. ${ }^{9,14-16}$

In our previous report, we proposed the onset threshold of the sFlt1/PlGF ratio using a manual enzyme immunosorbent assay (ELISA) for serum levels of sFlt-1 and PlGF and showed that women with an sFlt-1/PlGF ratio higher than the onset threshold at 26-31 weeks of gestation have a high risk of imminent onset of PE, although the detection rate of PE at 19-25 weeks was very poor. ${ }^{9}$ In the current

Department of Obstetrics and Gynecology, Jichi Medical University School of Medicine, Tochigi, Japan

Correspondence: Dr A Ohkuchi, Department of Obstetrics and Gynecology, Jichi Medical University School of Medicine, 3311-1 Yakushiji, Shimotsuke-shi, Tochigi 329-0498, Japan.

E-mail: okuchi@jichi.ac.jp

${ }^{1}$ These authors contributed equally to this work

Received 16 March 2013; revised 19 April 2013; accepted 19 May 2013; published online 22 August 2013 
study, we hypothesized that screening using the automated electrochemiluminescence immunoassay (ECLIA) for the plasma levels of sFlt-1 and PlGF would demonstrate similar performance to that of screening using ELISA, because the correlation determinations between the values determined by ECLIA and ELISA for serum/ plasma sFlt-1 and PlGF were shown to be sufficiently high (all $>0.7$; Ohkuchi et al. ${ }^{17}$ ) and because the sensitivity (SE) and specificity (SP) for diagnosing early-onset PE using the plasma levels of sFlt-1 and PlGF determined by ECLIA were shown to be $100 \% .{ }^{17}$ In addition, we thought that a rapid and fully automated method of measuring the circulating levels of sFlt-1 and PlGF using ECLIA would contribute to the early detection of PE.

In the current study, our first aim was to evaluate the onset threshold of the sFlt-1/PlGF ratio as determined by ECLIA for predicting the imminent onset of PE within 4 weeks after blood sampling at 19-25 and 26-31 weeks. Our second aim was to evaluate the use of plasma levels of sFlt-1 and PlGF as determined by ECLIA for predicting all PE; PE with onset at $<36,<34$ and $<32$ weeks of gestation; gestational hypertension $(\mathrm{GH})$; and small-for-gestationalage (SGA) infants.

\section{METHODS}

\section{Subjects}

We obtained written informed consent from all the women included in this study as well as approval from the Ethics Committee of our institute. Almost all study subjects were Japanese women with singleton pregnancies, and the subjects were divided into two groups. The first group consisted of 1724 unselected pregnant women who were recruited into a prospective cohort study in our tertiary center between April 2004 and September 2008. One hundred and twenty-three women were excluded, because their outcomes of $\mathrm{PE}$ or infant data could not be obtained. In the remaining 1601 women, PE occurred in 48 women (3.0\%), early-onset PE with onset at $<32$ weeks of gestation occurred in 16 women (1.0\%), GH occurred in 24 women (1.5\%) and an SGA infant occurred in 192 women (12.0\%). One thousand one hundred ninety-nine blood samples were collected at 19-25 weeks of gestation, and 798 blood samples were collected at 26-31 weeks of gestation. The second group consisted of 33 preeclamptic women from whom blood samples were drawn as soon as possible after admission to our hospital due to the clinical manifestation of PE between April 2004 and April 2007. Blood samples from some of the subjects in the first group and all of the subjects in the second group had been previously used to establish a gestational age-specific reference range of plasma levels of sFlt-1, PlGF and the sFlt-1/PlGF ratio and to evaluate the screening and diagnostic performances of those indices for the clinical manifestation of early- or late-onset PE. ${ }^{17,18}$

\section{Blood pressure in pregnancy and puerperal period}

Systolic blood pressure (SBP) and diastolic blood pressure (DBP) were measured using an Omron HEM-906 automated digital oscillometric sphygmomanometer (OMRON Healthcare, Kyoto, Japan) according to the standard procedures. The mean value of the two SBP and DBP readings at 16-19 and 20-23 weeks of gestation was documented for each participant.

\section{Measurement of uterine artery indices}

Color-pulsed Doppler ultrasound examination was performed using an SSD2200 (Aloka, Tokyo, Japan), an SSD-3500 (Aloka) or an SSD-5000 (Aloka) using a $3.5-\mathrm{MHz}$ transducer and a $50-\mathrm{Hz}$ high-pass filter. Details of the measurement of the uterine artery Doppler are included in the previous report. ${ }^{19}$ In brief, at least three waveforms from each side were viewed on the monitor. The peak systolic (a), end-diastolic (b), early diastolic (c) and maximum diastolic (d) Doppler velocities were measured using a built-in caliper, whereas the mean (m) Doppler velocity was automatically measured using a built-in automatic tracing system in two consecutive waveforms in each uterine artery. The pulsatility indices (PI) was defined as $(\mathrm{a}-\mathrm{b}) / \mathrm{m}$ and the notch depth index (NDI) was defined as $(\mathrm{d}-\mathrm{c}) / \mathrm{d}$ in the presence of notching or the NDI was equal to zero in the absence of notching. We defined notching as positive when a notch was observed in all the three consecutive waveforms. The average PI and NDI in two consecutive waveforms were calculated, and then the mPI and mNDI of both uterine arteries were calculated and used for analysis. When there were two recordings on different examination days, we used the earlier data. The inter-observer coefficient of variability for NDI was $6.9 \pm 3.9 \% .^{20}$ The normal reference values at $16-23$ weeks were determined in our previous study, ${ }^{19}$ and the cutoff values of $\mathrm{mNDI}$ and mPI for predicting $\mathrm{PE}$ were set at the 90th percentile in the distributions of mNDI and mPI at 16-23 weeks in normal pregnant women. ${ }^{19}$

\section{Measurement of plasma levels of sFlt-1 and PIGF}

Blood samples were collected in tubes containing EDTA, centrifuged at $4{ }^{\circ} \mathrm{C}$ at $1170 \times g$ for $15 \mathrm{~min}$, and stored at $-20^{\circ} \mathrm{C}$ until use. The ECLIAs of sFlt- 1 and PlGF using Elecsys sFlt-1 (Roche Diagnostics, Penzberg, Germany) and Elecsys PlGF (Roche Diagnostics) were performed using Modular Analytics E 170 (Roche Diagnostics) according to the manufacturer's instructions. The minimal detectable doses in the assays for sFlt-1 and PlGF are $15 \mathrm{pg} \mathrm{ml}^{-1}$ and $10 \mathrm{pg} \mathrm{ml}^{-1}$, respectively. The intra-assay and inter-assay coefficients of variation for sFlt- 1 are $0.5-6.8 \%$ and $0.7-11 \%$, respectively, and the intra-assay and inter-assay coefficients of variation for PlGF are $0.6-2.6 \%$ and $0.6-5.9 \%$, respectively (manufacturer's information). The plasma recovery rates of sFlt-1 and PIGF were 0.89 and 0.85 , respectively. ${ }^{17}$

Definition of cutoff points of PlGF, sFlt-1 and the sFlt-1/PlGF ratio Although we had determined the normal reference ranges of plasma levels of sFlt-1, PlGF and the sFlt-1/PlGF ratio during 20-38 weeks of gestation in our previous study, ${ }^{17}$ in the current study we decided to establish the 95th percentile of the plasma level of sFlt-1 $\left(\mathrm{pg} \mathrm{m}^{-1}\right)$ and the 5th percentile of the plasma level of PlGF $\left(\mathrm{pg} \mathrm{ml}^{-1}\right.$ ) at 19-25 and 26-31 weeks of gestation. We chose these percentiles, because we thought that simple cutoff values during 19-31 weeks, but not time-dependent cutoff values determined by quadric curves in our previous study, ${ }^{17}$ might facilitate the introduction of our method into clinical practice and because the number of normal pregnant women in the current study was almost 10 -fold greater than that in the previous study. ${ }^{17}$ The data of the logarithmic translations of sFlt-1 and PlGF at 19-25 weeks were almost normally distributed; therefore, we calculated the means and s.ds. of $\log _{10}$ sFlt-1 and $\log _{10}$ PlGF in 1155 normal pregnant women without GH or PE. Because the mean and s.d. of $\log _{10}$ sFlt-1 at $19-25$ weeks were 3.035 and 0.224 , respectively, the 95 th percentile of $\log _{10}$ sFlt- 1 was calculated as 3.404 using the equation mean $+1.645 \times$ s.d. Then, an abnormal sFlt- 1 ratio at $19-25$ weeks was defined as $\geqslant 2540$. Because the mean and s.d. of $\log _{10} \mathrm{PlGF}$ at $19-25$ weeks were 2.480 and 0.227 , respectively, the 5 th percentile of $\log _{10} \mathrm{PlGF}$ was calculated as 2.106 using the equation mean $=1.645 \times$ s.d. Then, an abnormal PIGF at 19-25 weeks was defined as $<128$. Similarly, the data of the logarithmic translation of sFlt-1 and PlGF at 26-31 weeks were almost normally distributed; therefore, we calculated the means and s.ds of $\log _{10} \mathrm{sFlt}-1$ and $\log _{10} \mathrm{PlGF}$ in 769 normal pregnant women without GH or PE. Because the mean and s.d. of $\log _{10}$ sFlt- 1 at $26-31$ weeks was 3.045 and 0.221 , respectively, the 95th percentile of $\log _{10}$ sFlt-1 was calculated as 3.408; thus an abnormal sFlt-1 at $26-31$ weeks was defined as $\geqslant 2560$. Because the mean and s.d. of $\log _{10} \mathrm{PlGF}$ at $26-31$ weeks was 2.706 and 0.277 , respectively, the 5th percentile of $\log _{10} \mathrm{PlGF}$ was calculated as 2.251; thus an abnormal PlGF at 26-31 weeks was defined as $<177$.

Likewise, an abnormal sFlt-1/PlGF ratio at 19-25 weeks was defined as $\geqslant 10.5$ and that at $26-31$ weeks was defined as $\geqslant 8.5$.

\section{Establishment of gestational age-specific onset thresholds of the plasma levels of the sFlt-1/PIGF ratio}

In our previous study, we reported that the standard deviation score (SDS) of $\log _{10}(\mathrm{sFlt}-1 / \mathrm{PlGF})$ determined by ELISA (R\&D Systems, Minneapolis, MN, USA) showed the highest correlation coefficient of -0.869 to the onset gestational weeks of PE, and thus we created the onset threshold of the sFlt-1/ PlGF ratio for predicting PE with onset at $<36$ weeks. ${ }^{9}$ Because we had already determined the levels of sFlt- 1 and PlGF and the sFlt-1/PlGF ratio in referred women with PE who had blood samples collected as soon as possible after 
admission to our hospital due to the clinical manifestation of PE between April 2004 and April 2007, ${ }^{17}$ we also established a gestational age-specific normal reference range curve for the plasma level of the sFlt-1/PlGF ratio in normal pregnant women at 19-38 weeks of gestation. ${ }^{17}$ We analyzed the correlation coefficients between the onset gestational weeks of PE and the logarithmictransformed raw values/SDSs of sFlt-1 and PlGF and the sFlt-1/PIGF ratio using 25 samples, which were taken within 1 week after the onset of PE. The SDS of $\log _{10}$ (sFlt-1/PlGF) showed the highest correlation $(-0.930)$, followed by the SDS of $\log _{10} \mathrm{PlGF}$ and the raw value of $\log _{10} \mathrm{PlGF}(0.922$ and 0.751 , respectively), whereas the SDS of $\log _{10} \mathrm{sFlt}-1$, the raw values of $\log _{10}(\mathrm{sFlt}-1 /$ PlGF $)$ and $\log _{10}$ sFlt- 1 were all $<0.7 \quad(-0.539,-0.196$ and -0.680 , respectively). Therefore, we judged that the onset threshold of the SDS of $\log _{10}($ sFlt-1/PlGF $)$ was the most predictive, using the biomarkers determined by ECLIA. We constructed a regression line between the onset gestational weeks of PE and the SDS of $\log _{10}$ (sFlt-1/PlGF) soon after onset and the lines for \pm 2.0 s.d., that is, the 2.5th and 97.5th percentile confidence lines (Figure 1a). We considered the 2.5th percentile line of $\log _{10}(\mathrm{sFlt}-1 / \mathrm{PlGF})$ as the onset threshold of PE, according to our previous method. ${ }^{9}$ The equation for the line representing the onset threshold of the sFlt-1/PlGF ratio was as follows:

Onset threshold of $\log _{10}(\mathrm{sFlt}-1 / \mathrm{PlGF})(\mathrm{SDS})=$

$-0.478 \times w+17.787$ (where $w=$ gestational weeks of the onset of PE).

Next, we transformed the equation using the gestational age-specific normal reference ranges of $\log _{10}$ (sFlt-1/PlGF) and the definition of SDS. ${ }^{17}$ Accordingly, the SDS was transformed to raw values:

$$
\begin{aligned}
& \text { Mean of the normal reference curve of } \log _{10} \mathrm{sFlt}-1 / \mathrm{PlGF}[\mathrm{m}] \\
& =0.00668 \times w^{2}-0.363 \times w+5.255 \text { (where } w=\text { gestational weeks at blood sampling). } \\
& \text { S.d. of the normal reference curve of } \log _{10} \mathrm{sFlt}-1 / \mathrm{PlGF}[\mathrm{SD}] \\
& =0.0116 \times w-0.00642 \text {. } \\
& \text { Raw value of onset threshold of } \log _{10}(\mathrm{sFlt}-1 / \mathrm{PlGF}) \\
& =\mathrm{SDS} \times \mathrm{SD}+m \\
& =(-0.487 \times w+17.787) \times(0.0116 \times w-0.00642)+0.00668 \times w^{2} \\
& -0.363 \times w+5.255 \\
& =0.00103 \times w^{2}-0.154 \times w+5.141 \text {. }
\end{aligned}
$$

Hence, the onset threshold of $\log _{10}(\mathrm{sFlt}-1 / \mathrm{PlGF})$ was converted from a linear function to a quadratic curve (Figure 1b).

\section{Establishment of gestational age-specific onset thresholds of the plasma levels of PIGF}

The details of the methods are included in the Supplementary Information.

\section{Definitions of PE, GH and an SGA Infant}

We defined PE and GH according to the definition and classification of pregnancy-induced hypertension (2004) of the Japan Society for the Study of Hypertension in Pregnancy. ${ }^{21}$ In brief, PE was defined as hypertension with

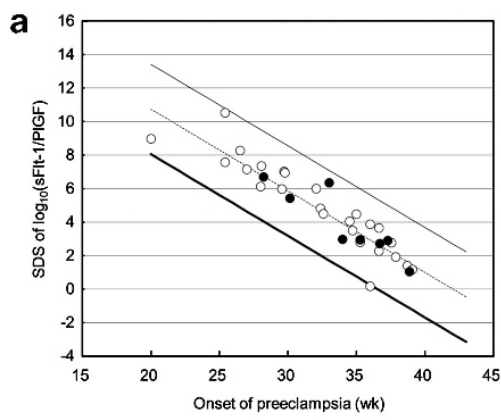

b
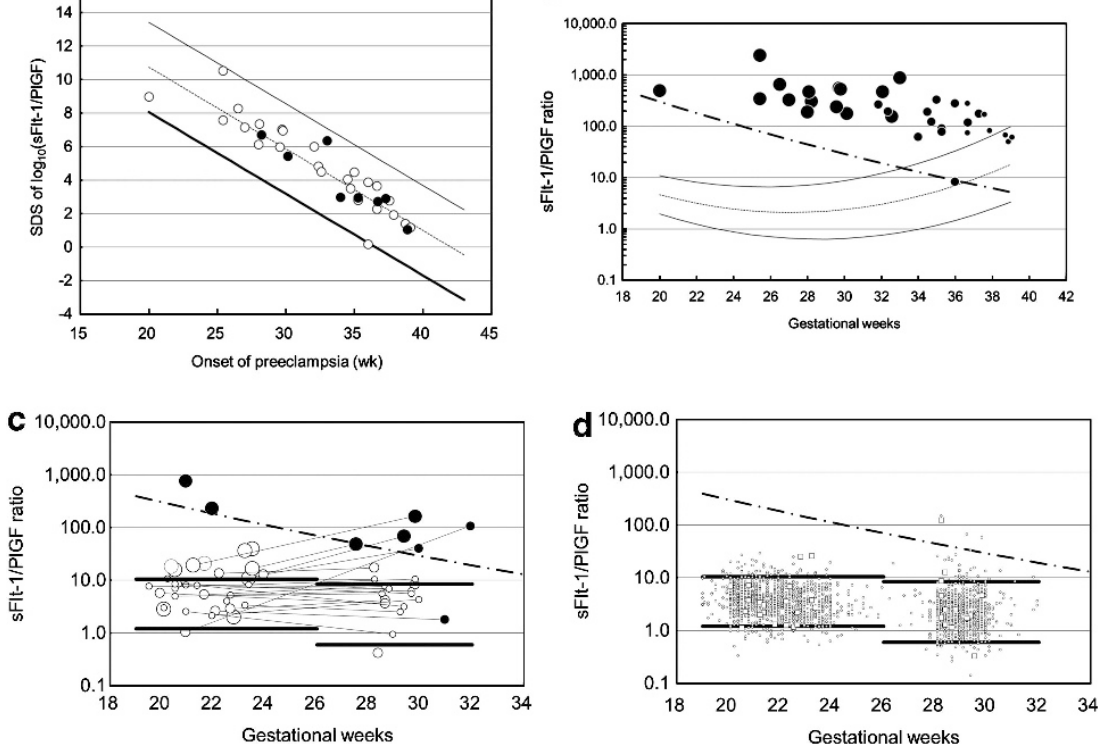

Figure 1 Prediction of the imminent onset of preeclampsia (PE) using the onset threshold of the soluble fms-like tyrosine kinase 1/placental growth factor (sFIt-1/PIGF) ratio. (a) We constructed regression lines between the onset gestational weeks of PE and the standard deviation scores (SDSs) of log 10 (sFlt-1/ PIGF) soon after onset and their 2.5th and 97.5th percentile confidence lines. The regression lines are represented by dotted lines, and the 2.5th and 95th percentile confidence lines are represented by straight lines. Thick straight lines represent the onset threshold. Open circles represent the SDSs at sampling $<7$ days after the onset of PE $(n=25)$, whereas closed circles represent the SDSs at sampling $\geqslant 7$ days after the onset of PE $(n=8)$. (b) The thin straight curves represent the 5th and 95th percentiles of the normal reference range of the sFIt-1/PIGF ratio, and the dotted curve represents the mean of the normal reference range of the sFIt-1/PIGF ratio. We drew the onset threshold of the plasma levels of the sFlt-1/PIGF ratio (dashed and dotted curve), which was the 2.5th percent confidence line of the regression line between the onset gestational weeks of PE and the SDSs of $\log _{10}(\mathrm{sFIt}-1 / \mathrm{PIGF})$ soon after onset. If the plasma levels of the sFIt-1/PIGF ratio were equal to or greater than the onset threshold of the sFIt-1/PIGF ratio, the sFIt-1/PIGF ratio was abnormal. The raw values of the sFIt-1/PIGF ratio after the onset of PE are shown. Large closed circles represent the values at sampling in preeclamptic women with onset at $<32$ weeks of gestation, medium closed circles represent those in preeclamptic women with onset between 32 and 35 weeks of gestation and small closed circles represent those in preeclamptic women with onset at $\geqslant 36$ weeks of gestation. (c, d) Thick straight lines represent the 5th and 95th percentiles of the distribution of $\log _{10}$ (sFIt-1/PIGF) in 1155 normal pregnant women at 19-25 weeks of gestation and 769 normal pregnant women at 26-31 weeks of gestation in a prospective cohort study. The dashed and dotted curve represents the onset threshold of the plasma levels of the sFlt-1/PIGF ratio. Large, medium and small circles (c) represent the values at sampling in preeclamptic women with onset at $<32,32-35$ and $\geqslant 36$ weeks of gestation, respectively; closed circles (c) represent cases of the imminent onset of PE within 4 weeks after blood sampling; and very small open circles (d) represent the values in normal pregnant women, whereas small open squares (d) represent the values in women with gestational hypertension. 
proteinuria occurring after 20 weeks of gestation. Superimposed PE was defined as chronic hypertension diagnosed before pregnancy or before 20 weeks of gestation, with proteinuria emerging afterward. Superimposed PE was included in the category of PE in this study. GH was defined as hypertension without proteinuria occurring after 20 weeks of gestation. Hypertension was defined as SBP $\geqslant 140 \mathrm{~mm} \mathrm{Hg}$ and/or DBP $\geqslant 90 \mathrm{~mm} \mathrm{Hg}$ on two occasions $\geqslant 4 \mathrm{~h}$ apart. Proteinuria was defined as $300 \mathrm{mgday}^{-1}$ from a 24-h urine collection. If only test tape was available, repeated semi-quantitative test results of $1+$, which represented $30 \mathrm{mg} \mathrm{dl}^{-1}$ protein or more, were considered to constitute a positive result. An SGA infant was defined as having a birth weight below the 10th percentile for the Japanese population. ${ }^{22}$

\section{Statistics}

We calculated the SDS as $(A-B) / C$ (where $A$ is the raw value, $B$ is the mean and $C$ is the s.d.). The results are presented as the mean \pm s.d. For plasma sFlt1, PlGF and the sFlt-1/PlGF ratio, statistical testing was conducted after logarithmic transformation. An unpaired $t$-test was used to compare two continuous variables. The chi-square test or Fisher's exact test were used to compare categorical data. The fitted curve/line estimation was performed using software included in the IBM SPSS software package (version 21; IBM, Armonk, NY, USA). The positive likelihood ratio (LR \pm ) and the $95 \%$ confidence interval were calculated using the Confidence Interval Calculator (created by Rob Herbert at the University of Sydney). ${ }^{23} \mathrm{~A} \mathrm{LR}+\geqslant 10,{ }^{24,25}$ a SE $\geqslant 0.8$ and a positive predictive value $(\mathrm{PPV}) \geqslant 0.20$ were considered clinically useful for screening. $P<0.05$ was considered statistically significant.

\section{RESULTS}

\section{Maternal and neonatal characteristics}

In women with $\mathrm{PE}$ in our cohorts, the frequency of women with a past history of $\mathrm{PE} / \mathrm{GH}$, a family history of hypertension, obesity, chronic hypertension, SBP at $16-23$ weeks $\geqslant 120 \mathrm{~mm} \mathrm{Hg}$, DBP at 16-23 weeks $\geqslant 80 \mathrm{~mm} \mathrm{Hg}, \mathrm{mPI}$ at $16-23$ weeks $\geqslant 90$ th percentile or mNDI at $16-23$ weeks $\geqslant 90$ th percentile was significantly higher than in controls, and the pre-pregnancy body mass index, SBP and DBP at 16-23 weeks were also significantly higher than in controls (Table 1). Women with PE delivered earlier and had smaller infants than controls.

\section{Risk factors for predicting the imminent onset of PE within 4 weeks after blood sampling}

The imminent onset of PE occurred within 4 weeks after blood sampling in 2 of $1199(0.2 \%)$ women recruited at 19-25 weeks and in 6 of $798(0.8 \%)$ women recruited at $26-31$ weeks. When we evaluated the screening performance for predicting the imminent onset of $\mathrm{PE}$ at 19-25 weeks using a single marker alone, the $L R+s$ of a past history of PE/GH, a family history of hypertension, obesity, SBP, DBP, mPI and mNDI were all $<10$; the LR $+s$ of the plasma levels of PlGF and sFlt- 1 and of the sFlt-1/PlGF ratio were all $\geqslant 10$. The corresponding SEs were all 1.00, but the PPVs were very low (Table 2). When we evaluated the screening performance for predicting the imminent onset of PE at 26-31 weeks using a single marker alone, the LR $+\mathrm{s}$ of the plasma levels of PlGF and sFlt-1 and of the sFlt-1/PlGF ratio were all $\geqslant 10$; the SEs were all $\geqslant 0.80$, but the PPVs were $<0.20$ (Table 3 ).

\section{Onset threshold of sFlt-1/PIGF ratio for predicting the imminent onset of PE within 4 weeks after blood sampling}

We evaluated the screening performance for predicting the imminent onset of PE within 4 weeks after blood sampling at 19-25 and 26-31 weeks using the onset threshold of plasma levels of the sFlt-1/PlGF ratio.

The onset threshold of plasma levels of the sFlt-1/PlGF ratio at 19-25 weeks showed an SE of 1.00, a LR + of $\infty$ and a PPV of 1.00 (Table 2). Thus, the onset threshold of plasma levels of the sFlt-1/ PlGF ratio at 19-25 weeks as determined by ECLIA was clinically
Table 1 Maternal and neonatal characteristics in normal pregnant women and preeclamptic women

\begin{tabular}{|c|c|c|c|c|}
\hline Characteristics & $\begin{array}{c}\text { Normal } \\
\text { pregnant } \\
\text { women }^{\mathrm{a}} \\
(\mathrm{n}=1529)\end{array}$ & $\begin{array}{l}\text { Women } \\
\text { with PE } \\
(\mathrm{n}=48)\end{array}$ & $\begin{array}{c}\text { Number } \\
\text { of } \\
\text { missing } \\
\text { values } \\
\text { (normal/ } \\
P E \text { ) }\end{array}$ & P-value \\
\hline \multicolumn{5}{|l|}{ Maternal } \\
\hline Age (mean \pm s.d., years) & $32.7 \pm 5.0$ & $33.7 \pm 5.5$ & 0 & 0.176 \\
\hline Age $\geqslant 40$ years $(\%)$ & $104(6.8)$ & $4(8.3)$ & 0 & 0.566 \\
\hline Race: Japanese (\%) & 1514 (99) & $48(100)$ & 0 & 1.000 \\
\hline Nulliparous women (\%) & $714(47)$ & $28(58)$ & 0 & 0.141 \\
\hline $\begin{array}{l}\text { Women with past history } \\
\text { of } \mathrm{PE} / \mathrm{GH}(\%)\end{array}$ & $57(3.7)$ & $9(19)$ & $2 / 1$ & $<0.001$ \\
\hline $\begin{array}{l}\text { Women with family history of } \\
\text { hypertension (\%) }\end{array}$ & $382(25)$ & $22(47)$ & $25 / 1$ & 0.002 \\
\hline Current smoker (\%) & $80(5.6)$ & $4(8.7)$ & $99 / 2$ & 0.328 \\
\hline $\begin{array}{l}\text { Pre-pregnancy BMI } \\
\left(\text { mean } \pm \text { s.d., } \mathrm{kg} \mathrm{m}^{-2}\right)^{\mathrm{b}}\end{array}$ & $22.2 \pm 4.1$ & $24.8 \pm 5.7$ & $5 / 0$ & 0.003 \\
\hline Obesity (\%) & $276(18)$ & $20(42)$ & $5 / 0$ & $<0.001$ \\
\hline Chronic hypertension (\%) & $22(1.4)$ & $13(27)$ & 0 & $<0.001$ \\
\hline $\begin{array}{l}\text { SBP at } 16-23 \text { wk } \\
\text { (mean } \pm \text { s.d., } \mathrm{mm} \mathrm{Hg} \text { ) }\end{array}$ & $116 \pm 14$ & $134 \pm 18$ & $5 / 1$ & $<0.001$ \\
\hline $\begin{array}{l}\text { DBP at } 16-23 \mathrm{wk} \\
\text { (mean } \pm \text { s.d., } \mathrm{mm} \mathrm{Hg} \text { ) }\end{array}$ & $67 \pm 9$ & $81 \pm 12$ & $5 / 1$ & $<0.001$ \\
\hline $\begin{array}{l}\text { SBP at } 16-23 w k \\
\geqslant 120 \mathrm{~mm} \mathrm{Hg}(\%)\end{array}$ & $538(35)$ & $38(81)$ & $5 / 1$ & $<0.001$ \\
\hline DBP at $16-23 w k \geqslant 80 \mathrm{~mm} \mathrm{Hg}(\%)$ & $143(9.4)$ & $23(49)$ & $5 / 1$ & $<0.001$ \\
\hline $\begin{array}{l}\mathrm{mPI} \text { at } 16-23 \text { wk } \geqslant 90 \text { th } \\
\text { percentile }(\%)^{\mathrm{b}}\end{array}$ & $185(13)$ & $21(45)$ & $63 / 1$ & $<0.001$ \\
\hline $\begin{array}{l}\mathrm{mNDI} \text { at } 16-23 \mathrm{wk} \\
\geqslant 90 \text { th percentile }(\%)^{\mathrm{b}}\end{array}$ & $162(11)$ & $19(40)$ & $63 / 1$ & $<0.001$ \\
\hline
\end{tabular}

\section{Neonatal}

Gestational age at delivery

$38.8 \pm 2.1 \quad 35.8 \pm 4.5 \quad 0 \quad<0.001$

(mean \pm s.d., wk)

Preterm delivery (\%)

$151(9.9) \quad 22(46) \quad 0 \quad<0.001$

Birthweight (mean \pm s.d., g)

Small-for-gestational-age

$2929 \pm 4862257 \pm 895 \quad 0 \quad<0.001$

$168(11) \quad 18(38) \quad 0 \quad<0.001$

infant $(\%)^{c}$

Abbreviations: BMI, body mass index; DBP, diastolic blood pressure; GH, gestational hypertension; mNDI, mean notch depth index; $\mathrm{mPI}$, mean pulsatility index;

$\mathrm{PE}$, preeclampsia; $\mathrm{SBP}$, systolic blood pressure; wk, weeks of gestation.

aNormal pregnant women were defined as women not complicated with PE or GH.

${ }^{\mathrm{b}}$ The cutoff values of 90th percentiles of $\mathrm{mPI}$ and $\mathrm{mNDI}$ were selected according to the respective normal reference value in our previous paper.

${ }^{c}$ A small-for-gestational-age infant was defined as an infant having a birth weight below the 10 th percentile for the Japanese population.

useful for predicting the imminent onset of PE at 19-25 weeks. Figure 1c shows that the sFlt-1/PlGF ratio in two women with an imminent onset of PE markedly deviated from the normal reference range of the sFlt-1/PlGF ratio at 19-25 weeks, and Figure 1d shows that all of the sFlt-1/PlGF ratios at 19-25 weeks in normal pregnant women or those with $\mathrm{GH}$ were located much lower than the onset threshold line of the sFlt-1/PlGF ratio.

The onset threshold of plasma levels of the sFlt-1/PlGF ratio at 26-31 weeks fulfilled three criteria of clinically useful screening: a $\mathrm{LR}+\geqslant 10$, an $\mathrm{SE} \geqslant 0.80$, and a PPV $\geqslant 0.20$ (Table 3 ). Figure $1 \mathrm{c}$ shows that the sFlt-1/PlGF level in $83 \%$ of women with an imminent onset of PE markedly deviated from the normal reference range of the 
Table 2 Screening performances for predicting imminent onset of PE within 4 wk after blood sampling, by single marker alone, and the onset thresholds of sFIt-1/PIGF ratio and PIGF, in 1199 women at 19-25 wk

\begin{tabular}{|c|c|c|c|c|c|c|c|c|c|c|c|}
\hline Risk factors & $\begin{array}{c}\text { Abnormal } \\
\text { value }\end{array}$ & $T P$ & $F N$ & FP & $T N$ & $S E$ & $S P$ & $L R+(95 \% \mathrm{Cl})$ & $L R-(95 \% \mathrm{Cl})$ & $P P V$ & NPV \\
\hline \multicolumn{12}{|l|}{ Single marker alone } \\
\hline Family history of hypertension & $(+)$ & 1 & 1 & 292 & 890 & 0.50 & 0.75 & $2.0(0.5-8.1)$ & $0.66(0.17-2.7)$ & 0.003 & 0.999 \\
\hline Obesity & $(+)$ & 1 & 1 & 225 & 968 & 0.50 & 0.81 & $2.7(0.7-11)$ & $0.62(0.15-2.5)$ & 0.004 & 0.999 \\
\hline SBP at $16-23$ wk & $\geqslant 120$ & 2 & 0 & 430 & 761 & 1.00 & 0.64 & $2.8(2.6-3.0)$ & 0 & 0.005 & 1.000 \\
\hline $\mathrm{mNDI}$ at $16-23 \mathrm{wk}$ & $\geqslant 90$ th & 1 & 1 & 143 & 1013 & 0.50 & 0.88 & $4.0(1.00-16)$ & $0.57(0.14-2.3)$ & 0.007 & 0.999 \\
\hline Plasma level of PIGF at $19-25$ wk & $<5.0$ th & 2 & 0 & 67 & 1130 & 1.00 & 0.94 & $18(14-23)$ & 0 & 0.029 & 1.000 \\
\hline Plasma level of sFlt-1 at $19-25$ wk & $\geqslant 95$ th & 2 & 0 & 66 & 1131 & 1.00 & 0.95 & $18(14-23)$ & 0 & 0.029 & 1.000 \\
\hline Plasma level of sFlt-1/PIGF ratio at $19-25$ wk & $\geqslant 95$ th & 2 & 0 & 70 & 1127 & 1.00 & 0.94 & $17(14-21)$ & 0 & 0.028 & 1.000 \\
\hline
\end{tabular}

Abbreviations: $\mathrm{Cl}$, confidence interval; DBP, diastolic blood pressure; FN, false negative; FP, false positive; GH, gestational hypertension; LR -, negative likelihood ratio; LR +, positive likelihood ratio; mNDI, mean notch depth index; mPI, mean pulsatility index; NPV, negative predictive value; PE, preeclampsia; PIGF, placental growth factor; PPV, positive predictive value; SBP, systolic blood pressure; sFIt-1, soluble fms-like tyrosine kinase 1; SE, sensitivity; SP, specificity; TN, true negative; TP, true positive; wk, weeks of gestation.

Bold font of LR + indicates that LR + was $\geqslant 10.0$

Bold font of PPV indicates that PPV was $\geqslant 0.20$

Table 3 Screening performances for imminent onset of PE within 4 wk after blood sampling, by single marker alone, and the onset thresholds of sFIt-1/PIGF ratio and PIGF, in 798 women at 26-31 wk

\begin{tabular}{|c|c|c|c|c|c|c|c|c|c|c|c|}
\hline Risk factors & $\begin{array}{c}\text { Abnormal } \\
\text { value }\end{array}$ & $T P$ & $F N$ & $F P$ & $T N$ & $S E$ & $S P$ & $L R+(95 \% C l)$ & $L R-(95 \% C l)$ & PPV & NPV \\
\hline \multicolumn{12}{|l|}{ Single marker alone } \\
\hline Women with past history of PE/GH & $(+)$ & 3 & 3 & 31 & 761 & 0.50 & 0.96 & $13(5.3-31)$ & $0.52(0.23-1.2)$ & 0.088 & 0.996 \\
\hline Family history of hypertension & $(+)$ & 3 & 3 & 188 & 591 & 0.50 & 0.76 & $2.1(0.9-4.7)$ & $0.66(030-1.5)$ & 0.016 & 0.995 \\
\hline Obesity & $(+)$ & 3 & 3 & 160 & 630 & 0.50 & 0.80 & $2.5(1.1-5.6)$ & $0.63(0.28-1.4)$ & 0.018 & 0.995 \\
\hline SBP at $16-23 w k$ & $\geqslant 120$ & 4 & 2 & 284 & 504 & 0.67 & 0.64 & $1.9(1.04-3.3)$ & $0.52(0.17-1.6)$ & 0.014 & 0.996 \\
\hline DBP at $16-23 w k$ & $\geqslant 80$ & 2 & 4 & 80 & 708 & 0.33 & 0.90 & $3.3(1.04-10)$ & $0.74(0.42-1.3)$ & 0.024 & 0.994 \\
\hline $\mathrm{mPI}$ at $16-23 \mathrm{wk}$ & $\geqslant 90$ th & 4 & 2 & 103 & 655 & 0.67 & 0.86 & $4.9(2.7-8.9)$ & $0.39(0.12-1.2)$ & 0.037 & 0.997 \\
\hline $\mathrm{mNDI}$ at $16-23$ wk & $\geqslant 90$ th & 5 & 1 & 81 & 677 & 0.83 & 0.89 & $7.8(5.2-12)$ & $0.19(0.03-1.1)$ & 0.058 & 0.999 \\
\hline Plasma level of PIGF at 26-31 wk & $<5$ th & 5 & 1 & 48 & 744 & 0.83 & 0.94 & $14(8.8-22)$ & $0.18(0.03-1.06)$ & 0.094 & 0.999 \\
\hline Plasma level of sFlt-1 at $26-31$ wk & $\geqslant 95$ th & 5 & 1 & 39 & 753 & 0.83 & 0.95 & $17(11-27)$ & $0.18(0.03-1.05)$ & 0.114 & 0.999 \\
\hline Plasma level of sFIt-1/PIGF ratio at $26-31 \mathrm{wk}$ & $\geqslant 95$ th & 5 & 1 & 45 & 747 & 0.83 & 0.94 & $15(9.3-23)$ & $0.18(0.03-1.06)$ & 0.100 & 0.999 \\
\hline \multicolumn{12}{|l|}{ Onset threshold of angiogenesis-related factor } \\
\hline Onset threshold of sFIt-1/PIGF ratio & & 5 & 1 & 5 & 787 & 0.83 & 0.994 & $132(51-339)$ & $0.17(0.03-1.00)$ & 0.500 & 0.999 \\
\hline Onset thershold of PIGF & & 5 & 1 & 28 & 764 & 0.83 & 0.965 & $24(14-39)$ & $0.17(0.03-1.03)$ & 0.152 & 0.999 \\
\hline
\end{tabular}

Abbreviations: $\mathrm{Cl}$, confidence interval; DBP, diastolic blood pressure; FN, false negative; FP, false positive; GH, gestational hypertension; LR -, negative likelihood ratio; LR +, positive likelihood ratio; $\mathrm{mNDI}$, mean notch depth index; $\mathrm{mPI}$, mean pulsatility index; NPV, negative predictive value; PE, preeclampsia; PIGF, placental growth factor; PPV, positive predictive value; SBP, systolic blood pressure; sFIt-1, soluble fms-like tyrosine kinase 1; SE, sensitivity; SP, specificity; TN, true negative; TP, true positive; wk, weeks of gestation.

blood pressure; sFIt-1, soluble fms-like tyrosi

Bold font of $L R+$ indicates that $L R+$ was $\geqslant 10.0$

Bold font of PPV indicates that PPV was $\geqslant 0.20$

sFlt-1/PlGF ratio at 26-31 weeks, and Figure $1 \mathrm{~d}$ shows that only $0.6 \%$ of women without an imminent onset of PE (four normal pregnant women and one woman with GH with onset 4 weeks after sampling) had an abnormal sFlt-1/PlGF ratio.

Onset threshold of PIGF for predicting the imminent onset of PE within 4 weeks after blood sampling

The details of the results are included in the Supplementary Information.
Comparison of screening properties of angiogenesis-related factors at $19-25$ and $26-31$ weeks for predicting all PE; $\mathrm{PE}$ with onset at $<36,<34$ and $<32$ weeks; GH; and an SGA infant

To predict all PE and PE with onset at $<36,<34$ and $<32$ weeks using the plasma levels of PlGF of $<5$ th percentile at 19-25 weeks, PE with onset at $<36,<34$ and $<32$ weeks showed a LR + of $\geqslant 10, \mathrm{PE}$ with onset at $<32$ weeks showed an SE of $\geqslant 0.80$ and no outcomes showed PPV of $\geqslant 0.20$ (Table 4). As for the plasma levels of sFlt-1 of 
Table 4 Screening performances for predicting imminent onset of PE within 4 wk after blood sampling in all PE; PE with onset at $<36,<34$ and <32 wk; GH; and SGA in 1199 women at 19-25 wk

\begin{tabular}{|c|c|c|c|c|c|c|c|c|c|c|c|}
\hline \multicolumn{12}{|l|}{$P I G F<5$ th } \\
\hline all PE & 0.028 & 13 & 21 & 56 & 1109 & 0.38 & 0.95 & $8.0(4.8-13)$ & $0.65(0.50-0.85)$ & 0.188 & 0.981 \\
\hline PE with onset at $<34$ wk & 0.012 & 10 & 4 & 59 & 1126 & 0.71 & 0.95 & $14(9.5-22)$ & $0.30(0.13-0.69)$ & 0.145 & 0.996 \\
\hline PE with onset at $<32$ wk & 0.009 & 9 & 2 & 60 & 1128 & 0.82 & 0.95 & $16(11-24)$ & $0.19(0.06-0.67)$ & 0.130 & 0.998 \\
\hline $\mathrm{GH}$ & 0.017 & 3 & 17 & 66 & 1113 & 0.15 & 0.94 & $2.7(0.92-7.8)$ & $0.90(0.75-1.08)$ & 0.043 & 0.985 \\
\hline \multicolumn{12}{|l|}{ sFlt-1 $\geqslant 95$ th } \\
\hline all PE & 0.028 & 5 & 29 & 63 & 1102 & 0.15 & 0.95 & $2.7(1.2-6.3)$ & $0.90(0.78-1.04)$ & 0.074 & 0.974 \\
\hline PE with onset at $<36$ wk & 0.015 & 4 & 14 & 64 & 1117 & 0.22 & 0.95 & $4.1(1.7-10)$ & $0.82(0.64-1.05)$ & 0.059 & 0.988 \\
\hline PE with onset at $<34$ wk & 0.012 & 3 & 11 & 65 & 1120 & 0.21 & 0.95 & $3.9(1.4-11)$ & $0.83(0.63-1.09)$ & 0.044 & 0.990 \\
\hline PE with onset at $<32$ wk & 0.009 & 3 & 8 & 65 & 1123 & 0.27 & 0.95 & $5.0(1.8-13)$ & $0.77(0.54-1.11)$ & 0.044 & 0.993 \\
\hline $\mathrm{GH}$ & 0.017 & 2 & 18 & 66 & 1113 & 0.10 & 0.94 & $1.8(0.47-6.8)$ & $0.95(0.82-1.10)$ & 0.029 & 0.984 \\
\hline PE with onset at $<36$ wk & 0.015 & 11 & 7 & 61 & 1120 & 0.61 & 0.95 & $12(7.6-18)$ & $0.41(0.23-0.73)$ & 0.153 & 0.994 \\
\hline PE with onset at $<34$ wk & 0.012 & 10 & 4 & 62 & 1123 & 0.71 & 0.95 & $14(9.1-21)$ & $0.30(0.13-0.69)$ & 0.139 & 0.996 \\
\hline PE with onset at $<32$ wk & 0.009 & 9 & 2 & 63 & 1125 & 0.82 & 0.95 & $15(11-22)$ & $0.19(0.06-0.67)$ & 0.125 & 0.998 \\
\hline $\mathrm{GH}$ & 0.017 & 2 & 18 & 70 & 1109 & 0.10 & 0.94 & $1.7(0.44-6.4)$ & $0.96(0.83-1.11)$ & 0.028 & 0.984 \\
\hline SGA & 0.116 & 11 & 128 & 61 & 999 & 0.08 & 0.94 & $1.4(0.74-2.5)$ & $0.98(0.93-0.95)$ & 0.153 & 0.886 \\
\hline
\end{tabular}

Abbreviations: $\mathrm{Cl}$, confidence interval; DBP, diastolic blood pressure; FN, false negative; FP, false positive; GH, gestational hypertension; LR -, negative likelihood ratio; LR +, positive likelihood ratio; mNDI, mean notch depth index; mPI, mean pulsatility index; NPV, negative predictive value; PE, preeclampsia; PIGF, placental growth factor; PPV, positive predictive value; SBP, systolic blood pressure; SE, sensitivity; sFlt-1, soluble fms-like tyrosine kinase 1; SGA, small-for-gestational age; SP, specificity; TN, true negative; TP, true positive; wk, weeks of gestation. Bold font of SE indicates that SE was $\geqslant 0.8$.

Bold font of $L R+$ indicates that $L R+$ was $\geqslant 10.0$

Bold font of PPV indicates that PPV was $\geqslant 0.20$.

$\geqslant 95$ th percentile at 19-25 weeks, no outcomes fulfilled the criteria for clinically useful screening performance. As for the plasma levels of sFlt-1/PlGF ratio of $\geqslant 95$ th percentile at 19-25 weeks, PE with onset at $<36,<34$ and $<32$ weeks showed a LR + of $\geqslant 10$, PE with onset at $<32$ weeks showed an SE of $\geqslant 0.80$ and no outcomes showed a PPV of $\geqslant 0.20$.

To predict all PE and PE with onset at $<36,<34$ and $<32$ weeks using the plasma levels of PlGF of $<5$ th percentile at $26-31$ weeks, $\mathrm{PE}$ with onset at $<34$ and $<32$ weeks showed a LR + of $\geqslant 10$ and an SE of $\geqslant 0.80$ but no outcomes showed a PPV of $\geqslant 0.20$ (Table 5). As for the plasma levels of sFlt- 1 of $\geqslant 95$ th percentile at $26-31$ weeks, $\mathrm{PE}$ with onset at $<36,<34$ and $<32$ weeks showed a LR + of $\geqslant 10$, $\mathrm{PE}$ with onset at $<34$ and $<32$ weeks showed an SE of $\geqslant 0.80$ and no outcomes showed a PPV of $\geqslant 0.20$. As for the plasma levels of sFlt-1/ PlGF ratio of $\geqslant 95$ th percentile at $26-31$ weeks, PE with onset at $<34$ and $<32$ weeks showed a LR + of $\geqslant 10$ and an SE of $\geqslant 0.80$ but no outcomes showed a PPV of $\geqslant 0.20$.

In predicting GH and SGA infants, none of the angiogenesis-related markers at $19-25$ or $26-31$ weeks showed an SE $\geqslant 0.80$ or a $L R+$ $\geqslant 10$ (Tables 4 and 5).

Comparison of receiver operating characteristics (ROC) curves of angiogenesis-related factors at 19-25 and 26-31 weeks for predicting all PE; PE with onset at $<36,<34$ and $<32$ weeks; GH; and an SGA infant

The details of the results are included in the Supplementary Information.

\section{DISCUSSION}

In this study, we disclosed two new findings. First, the onset threshold of the sFlt-1/PlGF ratio at 19-25 and 26-31 weeks as determined by ECLIA showed not only a high SE and a high LR + but also high PPV for predicting the imminent onset of PE within 4 weeks after blood sampling, showing an SE of $\geqslant 0.83$, an LR + of $\geqslant 132$ and a PPV of $\geqslant 0.50$. Thus the onset threshold of the sFlt-1/PlGF ratio at 19-31 weeks may be clinically useful for detecting imminent risk for the onset of PE within 4 weeks after blood sampling. Second, the plasma levels of PlGF of $<5$ th percentile and an sFlt-1/PlGF ratio $\geqslant 95$ th percentile at $19-25$ weeks showed a high SE and a high LR + but low PPV for predicting the imminent onset of PE and PE with onset at $<32$ weeks. Plasma levels of PlGF of $<5$ th percentile, plasma levels of sFlt- 1 of $\geqslant 95$ th percentile and an sFlt-1/PlGF ratio of $\geqslant 95$ th percentile at 26-31 weeks also showed a high SE and a high LR + but low PPV for predicting the imminent onset of PE and PE with onset at $<34$ and $<32$ weeks. Thus, the cutoff levels of 5 th percentile of PlGF and 95th percentile of sFlt-1 at 19-31 weeks may be useful for screening for high risk for PE with onset at $<34$ weeks.

The onset threshold of the sFlt-1/PlGF ratio at 19-31 weeks as determined by ECLIA may be clinically useful for detecting imminent risk for the onset of PE within 4 weeks after blood sampling. In our previous report, we reported the onset threshold of the sFlt-1/PlGF ratio and showed that women with an sFlt-1/PlGF ratio higher than the onset threshold at 26-31 weeks have a high risk of imminent onset of PE with onset at $<36$ weeks: at $2.2 \pm 0.6$ weeks after blood sampling, the ratio showed an SE of 0.36; however, the detection rate of $\mathrm{PE}$ at $19-25$ weeks was very poor, with an $\mathrm{SE}=0 .{ }^{9}$ In the current 
Table 5 Screening performances for predicting imminent onset of PE within 4 wk after blood sampling in all PE; PE with onset at $<36,<34$ and <32 wk; GH; and SGA in 798 women at 26-31 wk

\begin{tabular}{|c|c|c|c|c|c|c|c|c|c|c|c|}
\hline \multicolumn{12}{|l|}{ PIGF $<5$ th } \\
\hline All PE & 0.028 & 7 & 15 & 46 & 730 & 0.32 & 0.94 & $5.4(2.7-11)$ & $0.73(0.55-0.97)$ & 0.132 & 0.980 \\
\hline PE with onset at $<34$ wk & 0.008 & 5 & 1 & 48 & 744 & 0.83 & 0.94 & $14(8.8-22)$ & $0.18(0.03-1.06)$ & 0.094 & 0.999 \\
\hline PE with onset at $<32$ wk & 0.004 & 3 & 0 & 50 & 745 & 1.00 & 0.94 & $16(12-21)$ & 0 & 0.057 & 1.000 \\
\hline $\mathrm{GH}$ & 0.016 & 2 & 11 & 51 & 734 & 0.15 & 0.94 & $2.4(0.64-8.7)$ & $0.91(0.72-1.14)$ & 0.038 & 0.985 \\
\hline \multicolumn{12}{|l|}{ sFlt-1 $\geqslant 95$ th } \\
\hline All PE & 0.028 & 7 & 15 & 37 & 739 & 0.32 & 0.95 & 6.7 (3.4-13) & $0.72(0.54-0.95)$ & 0.159 & 0.980 \\
\hline PE with onset at $<36$ wk & 0.015 & 6 & 5 & 38 & 749 & 0.55 & 0.95 & $11(6.1-21)$ & $0.48(0.25-0.91)$ & 0.136 & 0.993 \\
\hline PE with onset at $<34$ wk & 0.012 & 5 & 1 & 39 & 753 & 0.83 & 0.95 & $17(11-27)$ & $0.18(0.03-846)$ & 0.114 & 0.999 \\
\hline PE with onset at $<32$ wk & 0.009 & 3 & 0 & 41 & 754 & 1.00 & 0.95 & $19(14-26)$ & 0 & 0.068 & 1.000 \\
\hline All PE & 0.028 & 8 & 14 & 42 & 734 & 0.36 & 0.95 & $6.7(3.6-13)$ & $0.67(0.49-0.92)$ & 0.160 & 0.981 \\
\hline PE with onset at $<36$ wk & 0.015 & 6 & 5 & 44 & 743 & 0.55 & 0.94 & $9.8(5.3-18)$ & $0.48(0.25-0.92)$ & 0.120 & 0.993 \\
\hline PE with onset at $<34$ wk & 0.012 & 5 & 1 & 45 & 747 & 0.83 & 0.94 & $15(9.3-23)$ & $0.18(0.03-1.06)$ & 0.100 & 0.999 \\
\hline PE with onset at $<32$ wk & 0.009 & 3 & 0 & 47 & 748 & 1.00 & 0.94 & $17(13-22)$ & 0 & 0.060 & 1.000 \\
\hline $\mathrm{GH}$ & 0.016 & 3 & 10 & 47 & 738 & 0.23 & 0.94 & $3.9(1.4-11)$ & $0.82(0.61-1.10)$ & 0.060 & 0.987 \\
\hline SGA & 0.096 & 14 & 63 & 36 & 685 & 0.18 & 0.95 & $3.6(2.1-6.4)$ & $0.86(2.2-8.3)$ & 0.280 & 0.916 \\
\hline
\end{tabular}

Abbreviations: $\mathrm{Cl}$, confidence interval; DBP, diastolic blood pressure; FN, false negative; FP, false positive; GH, gestational hypertension; LR -, negative likelihood ratio; LR +, positive likelihood ratio; mNDI, mean notch depth index; mPI, mean pulsatility index; NPV, negative predictive value; PE, preeclampsia; PIGF, placental growth factor; PPV, positive predictive value; SBP, systolic blood pressure; SE, sensitivity; sFlt-1, soluble fms-like tyrosine kinase 1; SGA, small-for-gestational age; SP, specificity; TN, true negative; TP, true positive; wk, weeks of gestation.

Bold font of SE indicates that SE was $\geqslant 0.8$.

Bold font of $L R+$ indicates that $L R+$ was $\geqslant 10.0$

Bold font of PPV indicates that PPV was $\geqslant 0.20$

study, the SEs of the onset threshold of the sFlt-1/PlGF ratio at 19-25 and 26-31 weeks were sufficiently high (1.00 and 0.83 , respectively), showing an LR + of $>100$ and a PPV of $\geqslant 0.50$, indicating that the onset threshold of sFlt-1/PlGF as determined by ECLIA at 19-31 weeks may be a good biomarker for predicting the imminent onset of PE. Improvement of the SE using the onset threshold of the sFlt-1/ PlGF ratio in our current study appeared to be the result of the improved SE of PE by PlGF using ECLIA.

PlGF $<5$ th percentile and an sFlt-1/PlGF ratio $\geqslant 95$ th percentile at 19-25 weeks showed a high SE and a high LR + but low PPV for predicting the imminent onset of $\mathrm{PE}$ and $\mathrm{PE}$ with onset at $<32$ weeks. These results suggested that plasma levels of PlGF and the sFlt1/PlGF ratio at 19-25 weeks may be useful in the detection of women at high risk for not only the imminent onset of PE within 4 weeks but also a later onset of PE (after 4 weeks). The imminent onset of PE occurred in only two pregnant women with blood sampling at 19-25 weeks, but PE with onset at $<32$ weeks occurred in 11 women with blood sampling at 19-25 weeks. Therefore, we should consider that a decrease of PlGF plasma levels or an increase of the sFlt-1/PlGF ratio at 19-25 weeks indicates a high risk for the occurrence of early-onset $\mathrm{PE}$ with onset at $<32$ weeks. In our previous study, based on PlGF plasma levels $<2.5$ th percentile and an sFlt-1/PlGF ratio $\geqslant 97.5$ th percentile as determined by ELISA, the use of PlGF levels at 19-25 weeks for predicting PE with onset at $<32$ weeks showed an SE, SP and LR + of 44\%, 96\% and 12, respectively. Furthermore, using the sFlt-1/PlGF ratio at 19-25 weeks for predicting PE with onset at $<32$ weeks showed an SE, SP and LR + of 78\%, 92\% and 47, respectively. ${ }^{9}$ Kleinrouweler et al. ${ }^{16}$ reviewed the accuracy of PlGF and sFlt-1 detection in the serum or plasma of pregnant women with blood sampling before 30 weeks of gestation and before the clinical onset of PE; of the 15 studies on PlGF, one study performed at 24 weeks reported an outcome of early-onset $\mathrm{PE}$ with onset at $<32$ weeks, with an SE of $84.4 \%$ and an SP of $95 \%{ }^{26}$ Taken together, low PlGF levels and a high sFlt-1/PlGF ratio in the second trimester appear to be high-risk markers for early-onset PE.

Plasma levels of PlGF of $<5$ th percentile, plasma levels of sFlt-1 of $\geqslant 95$ th percentile and an sFlt- $1 / \mathrm{PlGF}$ ratio of $\geqslant 95$ th percentile at 26-31 weeks also showed a high SE and a high LR + but low PPV for predicting the imminent onset of PE and PE with onset at $<34$ and $<32$ weeks. However, when we checked the predicted women in a database, the five women detected with imminent onset within 4 weeks after blood sampling using the onset threshold of the sFlt-1/PlGF ratio at 26-31 weeks were identical to the five women detected with $\mathrm{PE}$ with onset at $<34$ weeks using the plasma levels of PlGF of $<5$ th percentile, the plasma levels of sFlt-1 of $\geqslant 95$ th percentile and an sFlt-1/PlGF ratio of $\geqslant 95$ th percentile at $26-31$ weeks. Although the sFlt-1/PlGF ratio of $\geqslant 95$ th percentile at 26-31 weeks detected three other women with a later onset of PE after 34 weeks, the LR + decreased from 15 for the prediction of PE with onset at $<34$ weeks to 6.7 for the prediction of all $\mathrm{PE}$, suggesting that the screening of PE using PlGF and sFlt-1 at 26-31 weeks may be useful for detecting PE with onset at $<34$ weeks, but not for detecting $\mathrm{PE}$ with onset at $\geqslant 34$ weeks.

We also evaluated the screening performance for predicting the imminent onset of PE within 4 weeks after blood sampling at 19-25 and 26-31 weeks using the onset threshold of plasma levels of PlGF. 
The onset threshold of PlGF at 19-25 weeks was clinically useful but that at 26-31 weeks showed low PPV, although the LR \pm was $\geqslant 10$ and the SE was $\geqslant 0.8$. Therefore, if we select only one biomarker for predicting the imminent onset of PE at 19-31 weeks, we should select the sFlt-1/PlGF ratio rather than PlGF.

When we evaluated the ROC curves of the plasma levels of sFlt-1 and of PlGF and the sFlt-1/PlGF ratio at 19-25 and 26-31 weeks for predicting all $\mathrm{PE}$ and $\mathrm{PE}$ with onset at $<36,<34$ and $<32$ weeks, the areas under the curve (AUCs) of the sFlt-1/PlGF ratio and PlGF at 19-25 weeks of gestation were more accurate for predicting PE with earlier onset; however, the AUC of sFlt-1 at 19-25 weeks of gestation was very weak, even for predicting PE with onset at $<32$ weeks of gestation. In contrast, the AUCs of the three indices at 26-31 weeks of gestation were more accurate for predicting PE with earlier onset, and the AUCs of the three indices for earlier onset PE were identical. Thus, the results using ROC curves were almost consistent with those using the arbitrary cutoff values of PlGF plasma levels $<5$ th percentile, sFlt1 plasma levels $\geqslant 95$ th percentile and an sFlt-1/PlGF ratio $\geqslant 95$ th percentile.

In this study, the onset threshold of plasma levels of the sFlt-1/PlGF ratio was a highly sensitive and a highly specific screening method for detecting the imminent onset of PE within 4 weeks after blood sampling at 19-31 weeks; however, the imminent onset of $\mathrm{PE}$ occurred in only 2 of the $1199(0.2 \%)$ women recruited at $19-25$ weeks and in only 6 of the $798(0.8 \%)$ women recruited at 26-31 weeks. Because we planned to draw blood samples at 20-23 and 28-29 weeks in our study design, the time between the two samplings was relatively long, almost 8 weeks, resulting in several missed outcomes of PE for predicting the imminent onset of PE within 4 weeks after blood sampling. We are now planning a new study protocol with blood sampling every 4 weeks from 20 to 35 weeks. In addition, because our sample numbers for the target outcomes were relatively small, our findings should be confirmed in a larger multicenter cohort study. In this study, we did not evaluate the effects of sEng on predicting the imminent onset of PE within 4 weeks after blood sampling. We are now preparing to measure the levels of sEng in all the subjects in our current study and to analyze the onset threshold of sEng for predicting the imminent onset of PE.

\section{CONFLICT OF INTEREST}

The authors declare no conflict of interest.

\section{ACKNOWLEDGEMENTS}

This work was supported by Grants-in-Aid (18591809, 24390383 and 24592482 to AO) from the Ministry of Education, Culture, Sports, Science and Technology in Japan. We greatly thank Roche Diagnostics for the kind gifts of the Elecsys sFlt-1 and Elecsys PlGF and for their assistance with the sFlt-1 and PlGF measurements.

1 Nagaya K, Fetters MD, Ishikawa M, Kubo T, Koyanagi T, Saito Y, Sameshima H, Sugimoto M, Takagi K, Chiba Y, Honda H, Mukubo M, Kawamura M, Satoh S, Neki R. Causes of maternal mortality in Japan. JAMA 2000; 283: 2661-2667.

2 Yamauchi A, Minakami H, Ohkuchi A, Usui R, Idei S, Sato I. Causes of stillbirth: an analysis of 77 cases. J Obstet Gynaecol Res 1999; 25: 419-424.

3 Shiozaki A, Matsuda Y, Satoh S, Saito S. Impact of fetal sex in pregnancy-induced hypertension and $\geqslant$ in Japan. J Reprod Immunol 2011; 89: 133-139.

4 Levine RJ, Maynard SE, Qian C, Lim KH, England LJ, Yu KF, Schisterman EF, Thadhani R, Sachs BP, Epstein FH, Sibai BM, Sukhatme VP, Karumanchi SA
Circulating angiogenic factors and the risk of preeclampsia. N Eng/ J Med 2004; 350: 672-683

5 Levine RJ, Lam C, Qian C, Yu KF, Maynard SE, Sachs BP, Sibai BM, Epstein FH, Romero R, Thadhani R, Karumanchi SA, CPEP Study Group. Soluble endoglin and other circulating antiangiogenic factors in preeclampsia. N Eng/ J Med 2006; 355 992-1005.

6 Lim JH, Kim SY, Park SY, Yang JH, Kim MY, Ryu HM. Effective prediction of preeclampsia by a combined ratio of angiogenesis-related factors. Obstet Gynecol 2008; 111: 1403-1409.

7 Noori M, Donald AE, Angelakopoulou A, Hingorani AD, Williams DJ. Prospective study of placental angiogenic factors and maternal vascular function before and after preeclampsia and gestational hypertension. Circulation 2010; 122: 478-487.

8 Banek CT, Gilbert JS. Approaching the threshold for predicting preeclampsia: monitoring angiogenic balance during pregnancy. Hypertension 2011; 58: 774-775.

9 Ohkuchi A, Hirashima C, Matsubara S, Takahashi K, Matsuda Y, Suzuki M. Threshold of soluble fms-like tyrosine kinase 1/placental growth factor ratio for the imminent onset of preeclampsia. Hypertension 2011; 58: 859-866.

10 Maynard SE, Min JY, Merchan J, Lim KH, Li J, Mondal S, Libermann TA, Morgan JP, Sellke FW, Stillman IE, Epstein FH, Sukhatme VP, Karumanchi SA. Excess placenta soluble fms-like tyrosine kinase 1 (sFlt1) may contribute to endothelial dysfunction, hypertension, and proteinuria in preeclampsia. J Clin Invest 2003; 111: 649-658.

11 Venkatesha S, Toporsian M, Lam C, Hanai J, Mammoto T, Kim YM, Bdolah Y, Lim KH Yuan HT, Libermann TA, Stillman IE, Roberts D, D'Amore PA, Epstein FH, Sellke FW, Romero R, Sukhatme VP, Letarte M, Karumanchi SA. Soluble endoglin contributes to the pathogenesis of preeclampsia. Nat Med 2006; 12: 642-649.

12 Suzuki H, Ohkuchi A, Matsubara S, Takei Y, Murakami M, Shibuya M, Suzuki M, Sato Y. Effect of recombinant placental growth factor 2 on hypertension induced by full-length mouse soluble fms-like tyrosine kinase 1 adenoviral vector in pregnant mice. Hypertension 2009; 54: 1129-1135.

13 Kumasawa K, Ikawa M, Kidoya H, Hasuwa H, Saito-Fujita T, Morioka Y, Takakura N, Kimura T, Okabe M. Pravastatin induces placental growth factor (PGF) and ameliorates preeclampsia in a mouse model. Proc Natl Acad Sci USA 2011; 108: 1451-1455.

14 Cnossen JS, Morris RK, ter Riet G, Mol BW, van der Post JA, Coomarasamy A, Zwinderman AH, Robson SC, Bindels PJ, Kleijnen J, Khan KS. Use of uterine artery Doppler ultrasonography to predict pre-eclampsia and intrauterine growth restriction: a systematic review and bivariable meta-analysis. CMAJ 2008; 178: 701-711.

15 Cnossen JS, Vollebregt KC, de Vrieze N, ter Riet G, Mol BW, Franx A, Khan KS van der Post JA. Accuracy of mean arterial pressure and blood pressure measurements in predicting pre-eclampsia: systematic review and meta-analysis. BMJ 2008; 336 1117-1120.

16 Kleinrouweler CE, Wiegerinck MM, Ris-Stalpers C, Bossuyt PM, van der Post JA von Dadelszen $\mathrm{P}$, Mol BW, Pajkrt E, EBM CONNECT Collaboration. Accuracy of circulating placental growth factor, vascular endothelial growth factor, soluble fms-like tyrosine kinase 1 and soluble endoglin in the prediction of pre-eclampsia: a systematic review and meta-analysis. BJOG 2012; 119: 778-787.

17 Ohkuchi A, Hirashima C, Suzuki H, Takahashi K, Yoshida M, Matsubara S, Suzuki M. Evaluation of a new and automated electrochemiluminescence immunoassay for plasma sFIt-1 and PIGF levels in women with preeclampsia. Hypertens Res 2010; 33. 422-427.

18 Ohkuchi A, Ishibashi O, Hirashima C, Takahashi K, Matsubara S, Takizawa T, Suzuki M Plasma level of hydroxysteroid (17- $\beta$ ) dehydrogenase 1 in the second trimester is an independent risk factor for predicting preeclampsia after adjusting for the effects of mean blood pressure, bilateral notching and plasma level of soluble fms-like tyrosine kinase 1/placental growth factor ratio. Hypertens Res 2012; 35: 1152-1158.

19 Takahashi K, Ohkuchi A, Hirashima C, Matsubara S, Suzuki M. Establishing reference values for mean notch depth index, pulsatility index and resistance index in the uterine artery at 16-23 weeks' gestation. J Obstet Gynaecol Res 2012; 38: 1275-1285.

20 Ohkuchi A, Minakami H, Sato I, Mori H, Nakano T, Tateno M. Predicting the risk of preeclampsia and a small-for-gestational-age infant by quantitative assessment of the diastolic notch in uterine artery flow velocity waveforms in unselected women. Ultrasound Obstet Gynecol 2000; 16: 171-178.

21 Watanabe K, Naruse K, Tanaka K, Metoki H, Suzuki Y. Outline of definition and classification of "Pregnancy induced Hypertension (PIH)". Hypertens Res Pregnancy 2013; $1: 3-4$

22 Ogawa Y, Iwamura T, Kuriya N, Kuriya N, Nishida H, Takeuchi H, Yakada M, Itabashi K, Imura S, Isobe K. Birth size standards by gestational age for Japanese neonates. Acta Neonatol Jpn 1998; 34: 624-632.

23 Confidence interval calculator [Computer Program]. Available at http://www.pedro. org.au/english/downloads/confidence-interval-calculator/. Accessed 4 Mar 2012.

24 Jaeschke R, Guyatt G, Sackett DL. Users' guides to the medical literature. III. How to use an article about a diagnostic test. A. Are the results of the study valid? EvidenceBased Medicine Working Group. JAMA 1994; 271: 389-391.

25 Conde-Agudelo A, Villar J, Lindheimer M. World Health Organization systematic review of screening tests for preeclampsia. Obstet Gynecol 2004; 104: 1367-1391.

26 Crispi F, Llurba E, Domínguez C, Martín-Gallán P, Cabero L, Gratacós E. Predictive value of angiogenic factors and uterine artery Doppler for early- versus late-onset preclampsia and intrauterine growth restriction. Ultrasound Obstet Gynecol 2008; 31 303-309.

Supplementary Information accompanies the paper on Hypertension Research website (http://www.nature.com/hr) 